\title{
Efeito de Doses Reduzidas de OXYfluorfen em Cultivares de ALGODOEIRO ${ }^{1}$
}

\author{
Effect of Reduced Oxyfluorfen Rates on Cotton Cultivars
}

\author{
YAMASHITA, O.M. ${ }^{2}$, MENDONÇA, F.S. ${ }^{3}$, ORSI, J.V.N. ${ }^{3}$, RESENDE, D.D. ${ }^{3}$, KAPPES, C. ${ }^{3}$ e \\ GUIMARÃES, S.C. ${ }^{4}$
}

\begin{abstract}
RESUMO - O agronegócio do algodão apresenta-se, no Brasil, como uma atividade de grande importância para a economia nacional, ocupando posição de destaque no processo produtivo do Estado do Mato Grosso. Para que a cultura possa expressar o máximo potencial produtivo, é necessária a adoção de técnicas de manejo e práticas agronômicas, visando o controle das plantas daninhas. A fim de que a lavoura se mantenha limpa e livre de matocompetição, o controle químico é o método mais utilizado. Os herbicidas registrados para algodão devem ser aplicados, quando do estabelecimento da cultura, em jato dirigido. O contato desses produtos, como o oxyfluorfen, nas folhas pode incorrer em fitointoxicação e prejuizos para o produtor. O objetivo do presente trabalho foi mensurar o potencial de danos provocados pelo oxyfluorfen, quando em contato com diferentes cultivares de algodoeiro em estádio inicial de desenvolvimento. Para isso, foram avaliados cinco cultivares, tratados com 90 e $180 \mathrm{~g} \mathrm{ha}^{-1}$ do herbicida, aplicado em plantas com 15 e 30 dias de emergência. Quanto às variáveis analisadas (fitointoxicação, altura de planta, número de folhas, massa seca de planta), foi possivel observar que não houve diferença entre cultivares e que as plantas menores foram mais afetadas que as mais velhas, havendo aumento dos danos à medida que as doses eram aumentadas.
\end{abstract}

Palavras-chave: Gossypium hirsutum, herbicida, fitointoxicação, deriva

\begin{abstract}
Cotton agribusiness is a very important economic activity in Brazil, occupying a prominent position in the productive process of the state of Mato Grosso. For cotton crop to express its maximum productive potential, it is necessary to adopt handling techniques and agronomic practices seeking the control of weeds. For farming to stay clean and free from weed competition, chemical control is the most frequently used method. The herbicides registered for cotton crop should be applied when the culture is established in driven jet. The contact of products, such as oxyfluorfen, on the leaves may incur in plant intoxication and losses to the farmer. The objective of this work was to evaluate the effects of oxyfluorfen on five cotton cultivars at the initial development phase. The cotton cultivars were treated with 90 and $180 \mathrm{~g} \mathrm{ha}^{-1}$ of the herbicide applied 15 and 30 days after sowing. For the analyzed variables (phytointoxication, plant height, number ofleaves, plant dry mass), no difference was found among the cotton cultivars, with the smaller plants suffering more than the older plants, leading to increased damages, as doses increased.
\end{abstract}

Keywords: Gossypium hirsutum, herbicide, phytointoxication, drift.

1 Recebido para publicação em 6.3.2008 e na forma revisada em 8.9.2008.

2 Engo - -Agr ${ }^{0}$, M.Sc., Professor da UNEMAT - Alta Floresta-MT; Doutorando em Agricultura Tropical pela UFMT, <yama@unemat.br>; ${ }^{3}$ Estudantes de Agronomia da UNEMAT - Campus Universitário de Alta Floresta-MT; ${ }^{4}$ Engo-Agr ${ }^{0}$, Dr., Professor do DFF/FAMEV/UFMT 78060-900, Cuiabá-MT.

Planta Daninha, Viçosa-MG, v. 26, n. 4, p. 917-921, 2008 


\section{INTRODUÇÃO}

O cultivo de algodão apresenta-se como uma das mais importantes e rentáveis atividades relacionadas ao agronegócio brasileiro, tendo em vista a grande demanda mundial por fibras naturais e óleo vegetal.

Para que a cultura do algodoeiro expresse o máximo do seu potencial produtivo, é necessário o controle de fatores limitantes, entre os quais se destacam aqueles decorrentes da presença de plantas infestantes nas áreas cultivadas.

A utilização de herbicidas no controle de plantas infestantes é uma prática que tem se expandido em diferentes ambientes de cultivo, sendo um dos métodos mais eficientes e, em muitos casos, o mais econômico. Como os herbicidas são produtos fitossanitários, a sua aplicação requer conhecimento técnico aprimorado e um conjunto de cuidados que deve acompanhar o seu uso.

A aplicação de herbicidas em condições inapropriadas pode gerar problemas relacionados à deriva, atingindo culturas vizinhas (Hemphill Jr. \& Montgomery, 1981; Eberlein \& Guttieri, 1994; Wall, 1994; Ramsdale \& Messersmith, 2001; Ellis \& Griffin, 2002; Lyon et al., 2003).

Dessa maneira, a deriva apresenta-se como um fator de importância no momento em que os herbicidas, aplicados em áreas vizinhas, atingem culturas não-alvo, provocando fitointoxicação e sérios prejuízos às espécies e ao meio ambiente (Hemphill Jr. \& Montgomery, 1981; Banks \& Schroeder, 2002).

O grau de injúria e os sintomas observados são afetados por vários fatores, além do próprio modo de ação do herbicida, incluindo o clima, a espécie, o estádio de desenvolvimento da planta e a dose do herbicida (Al-Khatib et al., 1992; Alves et al., 2000; Yamashita \& Guimarães, 2005).

O controle químico de plantas infestantes na cultura do algodoeiro é realizado com a aplicação de alguns herbicidas registrados para essa cultura, entre eles o oxyfluorfen, recomendado em pré-emergência ou em pós-emergência das plantas por meio de aplicação dirigida (Rodrigues \& Almeida, 2005).
Por ser um herbicida não-seletivo para a cultura do algodoeiro e esta apresentar considerável sensibilidade a herbicidas (Snipes et al., 1999; Lyon et al., 2003; Miller et al., 2004; Ellis \& Griffin, 2002; Constantin et al., 2007), torna-se importante a obtenção de informações que possibilitem quantificar os prejuízos decorrentes da deriva de oxyfluorfen no algodoeiro.

Este trabalho teve por objetivo avaliar o efeito da aplicação de subdoses do herbicida oxyfluorfen, simulando deriva, em cinco cultivares de algodoeiro.

\section{MATERIAL E MÉTODOS}

O experimento foi instalado em casa de vegetação do Departamento de Agronomia da UNEMAT, Alta Floresta-MT, no período compreendido entre janeiro e abril de 2007.

Foi estudado o efeito do herbicida oxyfluorfen a 12,5 e $25,0 \%$ da dose recomendada para a cultura do algodoeiro, que representa 90 e $180 \mathrm{~g} \mathrm{ha}^{-1}$ do ingrediente ativo (Rodrigues \& Almeida, 2005). Adicionalmente, foi avaliada a testemunha sem herbicida.

Avaliaram-se cinco cultivares de algodoeiro: $\mathrm{Cv}-5$, Coodetec 406, BRS-jatobá, Ita-90 e Delta opal. Para cada cultivar, foram realizadas duas semeaduras, espaçadas de 15 dias, de forma a obter, numa única condição ambiental de aplicação de herbicida, plantas com idades de 15 e 30 dias da emergência.

Cada unidade experimental foi representada por um vaso plástico com substrato de solo coletado em área de mata (Tabela 1), retirandose os primeiros $20 \mathrm{~cm}$. Foi realizada calagem do substrato quatro meses antes da semeadura, elevando a saturação de bases para $60 \%$ (Staut \& Kurihara, 2001). A adubação foi estabelecida em função da análise de solo, conforme recomendação de pesquisa para a região (Fundação MT, 2001).

O delineamento experimental utilizado foi o inteiramente casualizado, no esquema fatorial $5 \times 3 \times 2$ (cinco cultivares, três doses do herbicida e duas idades de planta), com três repetições.

O herbicida oxyfluorfen foi aplicado por meio de um pulverizador costal de pressão constante, provido de barra com um bico de 
Tabela 1 - Características químicas do substrato utilizado no ensaio de cultivares submetidos a tratamento com doses crescentes de oxyfluorfen. Alta Floresta-MT, 2007

\begin{tabular}{|c|c|c|c|c|c|c|c|}
\hline \multirow{2}{*}{$\mathrm{pH} \mathrm{H}_{2} \mathrm{O}$} & $\mathrm{MO}$ & $\mathrm{P}$ & $\mathrm{K}$ & $\mathrm{Ca}$ & $\mathrm{Mg}$ & $\mathrm{Al}$ & \multirow{2}{*}{$\mathrm{V} \%$} \\
\cline { 2 - 7 } & $\mathrm{g} \mathrm{dm}^{-3}$ & \multicolumn{2}{|c|}{$-----\mathrm{mg} \mathrm{dm}^{-3}----$} & \multicolumn{2}{|c|}{$-----\mathrm{cmol}_{\mathrm{c}} \mathrm{dm}^{-3}$------ } & \\
\hline 5,4 & 29 & 0,71 & 13 & 1,40 & 0,60 & 0,10 & 27,54 \\
\hline
\end{tabular}

jato plano APG 110.02. O equipamento foi operado com pressão de $2 \mathrm{kgf} \mathrm{cm}^{-2}$, empregandose água como diluente, sendo calibrado para aplicação de $200 \mathrm{~L} \mathrm{ha}^{-1}$ de volume de calda.

Aos 7, 14, 21, 28 e 35 dias após a aplicação do herbicida (DAA), foram realizadas avaliações visuais de fitointoxicação das plantas de algodão através de notas (zero para ausência de sintomas e 100 para morte da planta); foi avaliada a altura da planta (tomada do colo até o ápice da haste principal da planta) e realizouse a contagem do número de folhas de cada planta.

No momento da última avaliação, as plantas foram cuidadosamente retiradas dos vasos, lavadas em água corrente e acondicionadas em sacos de papel. Posteriormente, foram secas em estufa de circulação forçada de $\operatorname{ar}\left(65^{\circ} \mathrm{C}\right)$ até peso constante, para obtenção de massa seca de parte aérea e de raiz.

Os resultados foram submetidos à análise de variância e as médias comparadas a $5 \%$ de probabilidade pelo teste de Scott \& Knott.

\section{RESULTADOS E DISCUSSÃO}

Para a variável fitointoxicação, ocorreu interação entre dose e idade de planta em todas as avaliações (Tabela 2). O efeito visual de fitointoxicação de algodoeiro foi caracterizado pela formação de cloroses de tonalidade bronzeada nas folhas, tendendo para o marrom, já na primeira avaliação (7 DAA). Esses sintomas evoluíram para necrose da área atingida pela calda herbicida, confirmando informações relatadas por Duke et al. (1991).

Observou-se que plantas tratadas precocemente (15 dias) tiveram maiores danos fitotóxicos que plantas em estádio de desenvolvimento mais avançado (30 dias) nas doses testadas, em todas as avaliações. Esses resultados concordam com os encontrados por Lunkes (1996), que, avaliando aplicação de oxyfluorfen no feijoeiro, observou que plantas são mais tolerantes ao oxyfluorfen à medida que se avança no seu estádio de desenvolvimento, e Alves et al. (2000), que, avaliando o mesmo herbicida em milho, observaram aumento nos sintomas visuais e decréscimo da produção à medida que as doses eram aumentadas.

Resultados semelhantes foram obtidos por Constantin et al. (2007) em plantas de algodoeiro, que se mostraram mais sensiveis à deriva de 2,4-D no estádio F1 (início do florescimento) do que no estádio C 1 (90\% das plantas com maçãs formadas), indicando também maior suscetibilidade em plantas mais jovens.

Para altura de planta, doses de herbicida provocaram efeitos significativos a partir da primeira avaliação $(p<0,05)$, não havendo, entretanto, diferença entre 90 e $180 \mathrm{~g} \mathrm{ha}^{-1}$ de oxyfluorfen (Tabela 3).

Observou-se que ambas as doses testadas provocaram efeitos significativos nas plantas de algodoeiro em todas as datas de avaliação. Esses resultados mostram que doses superiores

Tabela 2 - Fitointoxicação visual em plantas de algodoeiro tratado com oxyfluorfen, em duas idades (15 e 30 dias após a emergência), avaliada aos 7, 14, 21, 28 e 35 dias após aplicação (DAA). Alta Floresta-MT, 2007

\begin{tabular}{|c|c|c|c|c|c|c|c|c|c|c|}
\hline \multirow{5}{*}{$\begin{array}{c}\text { Dose } \\
\left(\mathrm{g} \mathrm{ha}^{-1}\right)\end{array}$} & \multicolumn{10}{|c|}{ Época de avaliação (dia após a aplicação) } \\
\hline & \multicolumn{2}{|c|}{7} & \multicolumn{2}{|c|}{14} & \multicolumn{2}{|c|}{21} & \multicolumn{2}{|c|}{28} & \multicolumn{2}{|c|}{35} \\
\hline & \multicolumn{10}{|c|}{ Idade da planta (dias) } \\
\hline & 15 & 30 & 15 & 30 & 15 & 30 & 15 & 30 & 15 & 30 \\
\hline & \multicolumn{10}{|c|}{ Fitointoxicação (\%) } \\
\hline 0 & $0,0 \mathrm{Aa}$ & $0,0 \mathrm{Aa}$ & $0,0 \mathrm{Aa}$ & $0,0 \mathrm{Aa}$ & $0,0 \mathrm{Aa}$ & $0,0 \mathrm{Aa}$ & $0,0 \mathrm{Aa}$ & $0,0 \mathrm{Aa}$ & $0,0 \mathrm{Aa}$ & $0,0 \mathrm{Aa}$ \\
\hline 90 & $37,2 \mathrm{Bb}$ & $24,3 \mathrm{Ab}$ & $40,8 \mathrm{Bb}$ & $34,2 \mathrm{Ab}$ & $39,2 \mathrm{Bb}$ & $28,9 \mathrm{Ab}$ & $32,0 \mathrm{Bb}$ & $30,7 \mathrm{Ab}$ & $40,3 \mathrm{Bb}$ & $31,7 \mathrm{Ab}$ \\
\hline 180 & $51,9 \mathrm{Bc}$ & $40,2 \mathrm{Ac}$ & $52,7 \mathrm{Bc}$ & $40,8 \mathrm{Ac}$ & $50,6 \mathrm{Bc}$ & $39,2 \mathrm{Ac}$ & $46,3 \mathrm{Bc}$ & $38,4 \mathrm{Ac}$ & $46,5 \mathrm{Ac}$ & $43,0 \mathrm{Ac}$ \\
\hline
\end{tabular}

Médias seguidas de mesma letra, maiúscula na linha e minúscula na coluna, para cada época de aplicação, não diferem entre si pelo teste de Scott \& Knott a 5\%. 
Tabela 3 - Altura de plantas de algodoeiro tratado com doses crescentes de oxyfluorfen após 7, 14, 21,28 e 35 dias da aplicação Alta Floresta-MT, 2007

\begin{tabular}{|c|c|c|c|c|c|}
\hline \multirow{2}{*}{ Cultivar } & \multicolumn{5}{|c|}{ Dia após a aplicação } \\
\hline & 7 & 14 & 21 & 28 & 35 \\
\hline $\mathrm{Cv}-5$ & $16,67 \mathrm{a}$ & $17,87 \mathrm{a}$ & $21,85 \mathrm{a}$ & $23,96 \mathrm{a}$ & $29,19 \mathrm{a}$ \\
\hline Coodetec 406 & $14,58 \mathrm{a}$ & $15,29 \mathrm{a}$ & $19,35 \mathrm{a}$ & $21,88 \mathrm{a}$ & $26,25 \mathrm{a}$ \\
\hline BRS-Jatobá & $15,36 \mathrm{a}$ & $16,16 \mathrm{a}$ & $20,89 \mathrm{a}$ & $23,86 \mathrm{a}$ & $28,66 \mathrm{a}$ \\
\hline Ita-90 & $16,52 \mathrm{a}$ & $17,55 \mathrm{a}$ & $20,57 \mathrm{a}$ & $24,55 \mathrm{a}$ & $31,00 \mathrm{a}$ \\
\hline Delta Opal & $16,27 \mathrm{a}$ & $17,16 \mathrm{a}$ & $21,50 \mathrm{a}$ & $24,00 \mathrm{a}$ & $29,22 \mathrm{a}$ \\
\hline Valor de F & $0,814^{\mathrm{ns}}$ & $0,990^{\mathrm{ns}}$ & $0,838^{\mathrm{ns}}$ & $0,795^{\mathrm{ns}}$ & $1,440^{\mathrm{ns}}$ \\
\hline \multicolumn{6}{|l|}{ Dose $\left(\mathrm{g} \mathrm{ha}^{-1}\right)$} \\
\hline 0 & $17,54 \mathrm{a}$ & $19,17 \mathrm{a}$ & $24,01 \mathrm{a}$ & $28,13 \mathrm{a}$ & $34,23 \mathrm{a}$ \\
\hline 90 & $15,60 \mathrm{~b}$ & $16,16 \mathrm{~b}$ & $19,29 \mathrm{~b}$ & $21,07 \mathrm{~b}$ & $25,70 \mathrm{~b}$ \\
\hline 180 & $14,50 \mathrm{~b}$ & $15,09 \mathrm{~b}$ & $19,20 \mathrm{~b}$ & $21,75 \mathrm{~b}$ & $26,66 \mathrm{~b}$ \\
\hline Valor de F & $4,082 *$ & $6,552 *$ & $11,224^{*}$ & $19,229 *$ & $17,963 *$ \\
\hline Int. (dose $\mathrm{x}$ estádio) valor de $\mathrm{F}$ & $0,488^{\mathrm{ns}}$ & $0,560^{\mathrm{ns}}$ & $0,622^{\mathrm{ns}}$ & $0,839^{\mathrm{ns}}$ & $0,642^{\mathrm{ns}}$ \\
\hline $\mathrm{CV}(\%)$ & 26,32 & 26,97 & 21,59 & 20,56 & 20,92 \\
\hline
\end{tabular}

Médias seguidas da mesma letra, nas colunas, não diferem estatisticamente entre si pelo teste de Scott \& Knott $(\mathrm{p}<0,05)$.

* Médias significativas pelo teste $\mathrm{F}(\mathrm{p}<0,05)$.

a $90 \mathrm{~g} \mathrm{ha}^{-1}$ de oxyfluorfen são significativamente tóxicas para plantas de algodoeiro mesmo após 35 dias da sua aplicação.

Os cultivares testados apresentaram resposta à altura semelhante entre si. Esses resultados discordam dos obtidos por Yamashita $\&$ Guimarães (2005), os quais, testando a resposta de algodoeiro à aplicação de doses reduzidas de glyphosate, observaram resposta diferencial entre cultivares.

Quanto à variável número de folhas, houve significância para a interação entre dose e idade de planta $(\mathrm{p}<0,05)$ apenas aos 7,14 e 21 DAA (Tabela 4) e interação entre cultivar e idade de planta $(\mathrm{p}<0,05)$ aos 28 e 35 DAA (Tabela 5).
Aos 7 e 14 DAA, foi observado que o número de folhas de plantas jovens era inferior ao número de folhas de plantas mais velhas, independentemente da dose de herbicida. Aos 21 DAA, apenas plantas tratadas com $90 \mathrm{~g} \mathrm{ha}^{-1} \mathrm{de}$ oxyfluorfen apresentaram número semelhante de folhas em plantas com 15 e 30 dias de idade. Nos demais tratamentos (0 e $\left.180 \mathrm{~g} \mathrm{ha}^{-1}\right)$, o número de folhas foi maior em plantas com 30 dias (Tabela 4). Nesta mesma tabela, é possivel observar que a maior dose provocou redução no número de folhas até a avaliação realizada aos $21 \mathrm{DAA}$, para plantas tratadas precocemente, e apenas aos 7 DAA, para plantas tratadas com 30 dias. Aos 14 e 21 DAA, plantas velhas submetidas à aplicação de oxyfluorfen não diferenciaram entre si, apenas da testemunha. Esses resultados comprovam

Tabela 4 - Número médio de folhas de plantas de algodoeiro submetidas a subdoses de oxyfluorfen em duas idades (15 e 30 dias após a emergência), aos 7, 14 e 21 dias após aplicação (DAA). Alta Floresta-MT, 2007

\begin{tabular}{|c|c|c|c|c|c|c|}
\hline \multirow{2}{*}{$\begin{array}{c}\text { Dose } \\
\left(\mathrm{g} \mathrm{ha}^{-1}\right)\end{array}$} & \multicolumn{2}{|c|}{$7 \mathrm{DAA}$} & \multicolumn{2}{c|}{$14 \mathrm{DAA}$} & \multicolumn{2}{c|}{$21 \mathrm{DAA}$} \\
\cline { 2 - 7 } & 15 dias & $30 \mathrm{dias}$ & 15 dias & 30 dias & 15 dias & 30 dias \\
\hline 0 & $3,0 \mathrm{Ba}$ & $5,6 \mathrm{Aa}$ & $4,0 \mathrm{Ba}$ & $5,7 \mathrm{Aa}$ & $4,6 \mathrm{Ba}$ & $5,8 \mathrm{Aa}$ \\
\hline 90 & $1,5 \mathrm{Bb}$ & $4,3 \mathrm{Ab}$ & $2,0 \mathrm{Bb}$ & $3,7 \mathrm{Ab}$ & $3,5 \mathrm{Ab}$ & $3,6 \mathrm{Ab}$ \\
\hline 180 & $0,6 \mathrm{Bc}$ & $3,2 \mathrm{Ac}$ & $1,1 \mathrm{Bc}$ & $3,7 \mathrm{Ab}$ & $1,1 \mathrm{Bc}$ & $4,0 \mathrm{Ab}$ \\
\hline
\end{tabular}

Médias seguidas de mesma letra, maiúscula na linha e minúscula na coluna, para cada época de aplicação, não diferem entre si pelo teste de Scott \& Knott a $5 \%$. 
Tabela 5 - Número médio de folhas de cultivares de algodoeiro tratados com oxyfluorfen em duas idades de planta (15 e 30 dias após a emergência), aos 28 e 35 dias após aplicação. Alta Floresta-MT, 2007

\begin{tabular}{|c|c|c|c|c|}
\hline \multirow{2}{*}{ Cultivar } & \multicolumn{2}{|c|}{$28 \mathrm{DAA}$} & \multicolumn{2}{c|}{$35 \mathrm{DAA}$} \\
\cline { 2 - 5 } & 15 dias & 30 dias & 15 dias & 30 dias \\
\hline CV-5 & $3,7 \mathrm{bB}$ & $5,7 \mathrm{aA}$ & $5,0 \mathrm{bB}$ & $7,1 \mathrm{aA}$ \\
\hline BRS-Jatobá & $4,3 \mathrm{aB}$ & $5,4 \mathrm{aA}$ & $6,1 \mathrm{aA}$ & $6,9 \mathrm{aA}$ \\
\hline Delta Opal & $4,4 \mathrm{aB}$ & $5,3 \mathrm{aA}$ & $6,1 \mathrm{aB}$ & $7,3 \mathrm{aA}$ \\
\hline ITA-90 & $4,5 \mathrm{aA}$ & $5,2 \mathrm{aA}$ & $6,2 \mathrm{aA}$ & $6,9 \mathrm{aA}$ \\
\hline CD-406 & $4,9 \mathrm{aA}$ & $4,7 \mathrm{aA}$ & $6,4 \mathrm{aA}$ & $6,5 \mathrm{aA}$ \\
\hline
\end{tabular}

Médias seguidas de mesma letra, maiúscula na linha e minúscula na coluna, para cada época de aplicação, não diferem entre si pelo teste de Scott \& Knott a $5 \%$.

a tolerância ao herbicida quando as plantas se apresentam em estádio de desenvolvimento mais avançado, concordando com resultados obtidos por Lunkes (1996) em feijoeiro e Alves et al. (2000) em milho.

Os cultivares Ita-90 e CD-406 não diferiram no número de folhas nas avaliações aos 28 e 35 DAA, tanto em plantas jovens quanto nas velhas. Essa observação também foi constatada no cultivar BRS-jatobá aos 35 DAA (Tabela 5). A mesma tabela mostra que o cultivar $\mathrm{Cv}-5$ apresentou menor número de folhas em plantas mais jovens que em plantas mais velhas, em ambas as avaliações (28 e 35 DAA).

Nas condições dos ensaios, foi possivel concluir que, sempre que o herbicida causou algum tipo de prejuízo à cultura do algodoeiro, esses danos se deram de forma crescente, não havendo diferença entre os cultivares testados. Subdoses aplicadas em plantas mais jovens podem provocar danos significativos às plantas, possibilitando perdas na produtividade.

\section{LITERATURA CITADA}

AL-KHATIB, K.; PARKER, R.; FUERST, E. P. Sweet cherry (Prumus avium) response to simulated drift from selected herbicides. Weed Technol., v. 6, n. 6, p. 975-79, 1992.

ALVES, L. W. R.; SILVA, J. B.; SOUZA, I. F. Efeito da aplicação de subdoses dos herbicidas glyphosate e oxyfluorfen, simulando deriva sobre a cultura de milho (Zea mays L.). Ci. Agrotec., v. 24, n. 4, p. 889-897, 2000.
BANKS, P. A.; SCHROEDER, J. Carrier volume affects herbicide activity in simulated spray drift studies. Weed Technol., v. 16, n. 4, p. 833-837, 2002.

CONSTANTIN, J. et al. Efeito de subdoses de 2,4-D na produtividade do algodão e suscetibilidade da cultura em função de seu estádio de desenvolvimento. Eng. Agríc., v. 27, n 3, p.24-29, 2007.

DUKE, S. O. et al. Protoporphyrinogen oxidase-inhibiting herbicides. Weed Sci., v. 39, n. 3, p. 465-473, 1991.

EBERLEIN, C. V.; GUTTIERI, M. J. Potato (Potato tuberosum) response to simulate drift of imidazoline herbicides. Weed Sci., v. 42, n. 1, p. 70-75, 1994.

ELLIS, J. M.; GRIFFIN, J. Soybean (Glycine max) and cotton (Gossypium hirsutum) response to simulated drift of gyphosate and glyfosinate. Weed Technol., v. 16, n. 3, p. $580-586,2002$

LYON, L. L. et al. Non glyphosate tolerant cotton response to simulated drift rates glyphosate. Proc. South. Weed Sci. Soc., v.56, p.14-15, 2003.

LUNKES, J. A. Efeito de subdoses de glyphosate e oxyfluorfen simulando deriva sobre a cultura do feijoeiro. 1996. $138 \mathrm{f}$. Tese (Doutorado em Fitotecnia) Universidade Federal de Lavras, Lavras, 1996.

FUNDAÇÃO MT. Boletim de pesquisa de algodão. Rondonópolis: 2001. 225 p. (Boletim, 4).

HEMPHILL Jr., D. D.; MONTGOMERY, M. L. Response of vegetable crops to sublethal application of 2,4-D. Weed Sci., v. 29, n. 6, p. 632-635, 1981.

MILLER, D. K. et al. Response of non glyphosate resistant cotton to reduced rates of glyphosate. Weed Sci., v. 52, n. 1, p. 178-182, 2004

RAMSDALE, B. K.; MESSERSMITH, C. G. Drift-reducing nozzle effects on herbicide performance. Weed Technol., v. 15, n. 3, p. 453-460, 2001.

RODRIGUES, B. N.; ALMEIDA, F. S. Guia de herbicidas. 5.ed. Londrina: IAPAR, 2005. $591 \mathrm{p}$.

SNIPES, C. E.; STREET, J. E.; MUELLER, T. C. Cotton (Gossypium hirsutum) response to simulated quinclorac drift. Weed Sci., v. 40, n. 1, p. 106-109, 1999.

STAUT, L. A.; KURIHARA, C. H. Calagem e adubação. In: EMBRAPA AGROPECUÁRIA OESTE; EMBRAPA ALGODÃO. Algodão: tecnologia de produção. Dourados: 2001. p. 103-123.

WALL, D. A. Potato (Solanum tuberosum) response to simulated drift of dicamba, clopyralid, and tribenuron. Weed Sci., v. 42, n. 1, p. 110-114, 1994.

YAMASHITA, O. M.; GUIMARÃES, S. C. Resposta de cultivares de algodão a subdoses de glyphosate. Planta Daninha, v. 23, n. 4, p. 627-633, 2005. 